OPEN

SUBJECT AREAS:

COLLOIDS

STRUCTURE OF SOLIDS AND

LIQUIDS

STATISTICAL PHYSICS,

THERMODYNAMICS AND

NONLINEAR DYNAMICS

STRUCTURE OF SOLIDS AND

LIQUIDS

Received

26 September 2012

Accepted

3 December 2012

Published

21 December 2012

Correspondence and requests for materials should be addressed to U.S. (ullrich.siems@unikonstanz.de)

\section{Non-monotonic crossover from single-file to regular diffusion in micro-channels}

\author{
U. Siems' ${ }^{1}$, C. Kreuter' ${ }^{1}$ A. Erbe ${ }^{2}$, N. Schwierz ${ }^{3}$, S. Sengupta ${ }^{4,5}$, P. Leiderer' \& P. Nielaba'
}

'Department of Physics, University of Konstanz, 78457 Konstanz, Germany, ${ }^{2}$ Helmholtz-Center Dresden-Rossendorf, 01314 Dresden, Germany, ${ }^{3}$ Department of Physics, Technical University of Munich, 85748 Garching, Germany, ${ }^{4}$ TIFR Centre for Interdisciplinary Sciences, 21 Brundavan Colony, Narsingi, Hyderabad 500075, India, ${ }^{5}$ Centre for Advanced Materials, Indian Association for the Cultivation of Science, Jadavpur, Kolkata 700032, India.

The diffusion behavior of interacting particles determines the behavior of a large number of systems ranging from pedestrians crossing a road to ions passing through channels in living cells. Here we present a system in which the nature of the diffusion process varies with changes in the external conditions. We find this special behavior in a colloidal model system, consisting of micron sized particles which are confined to narrow channels and interact via induced magnetic dipoles. When the density of these particles is changed, diffusion alternates between normal Fickian behavior and single-file diffusion. This anomalous behavior is induced by the order of the particles in the restricted geometry and does not depend on the exact nature of the inter-particle interactions.

r he analysis of interesting non-equilibrium phenomena in confined geometries has become possible over the last few years, ranging from the dynamics of pedestrians in a pedestrian zone ${ }^{1}$ to the electron transport and thermopower effects in atomic wires ${ }^{2,3}$, the biologically important size selectivity of transport in ion channels $s^{4}$, the mixing in "lab-on-chip" devices ${ }^{5}$ and the pattern formation of colloids in micro-channels ${ }^{6,7}$ and electric fields ${ }^{8}$.

In this context, colloids have received much attention, since they often serve as model systems ${ }^{9-13}$ due to the ease of imaging particle coordinates by light scattering methods. The theoretical analysis of colloids has put forward the development of Brownian dynamics methods ${ }^{14-16}$.

Here we report on unexpected and important aspects of the diffusion of colloids in a confined geometry, comparing experiments with numerical simulations. Our work may have impact on many other fields, where the motion of individual particles is affected by constrictions, as mentioned above.

\title{
Results
}

Our system consists of colloidal particles confined to channels of width $L_{Y}$. The repulsive interaction between the particles at distance $r_{i j}$ can be described by a dipole potential $V_{i j}\left(r_{i j}\right)=\left(\mu_{0} / 4 \pi\right) M^{2} / r_{i j}^{3}$, with the magnetic dipole moments $M=\chi_{\text {eff }} B_{\text {ext }}$ of the particles ${ }^{18}$, where $B_{\text {ext }}$ represents the external applied magnetic field. Typical values for $\chi_{\text {eff }}$ are in the order of $7.8810^{-11} \mathrm{Am}^{2} / \mathrm{T}^{19}$. It is known that super-paramagnetic colloidal particles in twodimensional channels order into layers along the channel walls ${ }^{22}$. The ratio of potential to thermal energy is characterized by the parameter $\Gamma=\mu_{0} M^{2} /\left(4 \pi k_{B} T R^{3}\right)^{22}$, which can be interpreted as an inverse temperature of the system, where $R=(\sqrt{3} / 2 n)^{0.5}$ is the layer spacing of a triangular lattice with density $n$. We introduce reduced (dimensionless) length scales $A^{*}=A / \sigma$ of length scales $A$, e.g. $L_{Y}^{*}=L_{Y} / \sigma$, where the channel width $L_{Y}$ denotes the region, which is accessible for the particle centers, and $n^{*}=n \sigma^{2}$. Times are given in units of $\tau_{D}=\sigma^{2} / D_{0}$, where $D_{0}$ is the diffusion coefficient. Please note that $\tau_{D}=1$ corresponds to 218 seconds in the experiment.

We choose an interaction strength of $\Gamma \approx 13$, which is close to the crossover from a solid to a liquid phase. This is achieved by means of a fixed density of $n^{*}=0.4$ and a fixed external field of $B_{\text {ext }}=0.16 \mathrm{mT}$. In this case the structure inside the channel is only a function of the channel width $L_{Y}$. If the width is varied, the system switches between layering order and a more disordered state. In Figure 1 a snapshot of such equilibrium configurations is shown. For channels of width $L_{Y}^{*}=3.9$ and $L_{Y}^{*}=5.3$, the particles are ordered in three and four layers, respectively. For $L_{Y}^{*}=4.5$, the crossover from three to four layers takes place.

The main goal of this work is to study the influence of this layered structure on particle diffusion. For channels with a width $L_{Y}^{*}=1$ (see Figure 2), single-file diffusion is found, similar to a one dimensional channel, since the mutual passing of particles is forbidden due to hard-core interaction. On very short time scales, the mean square 

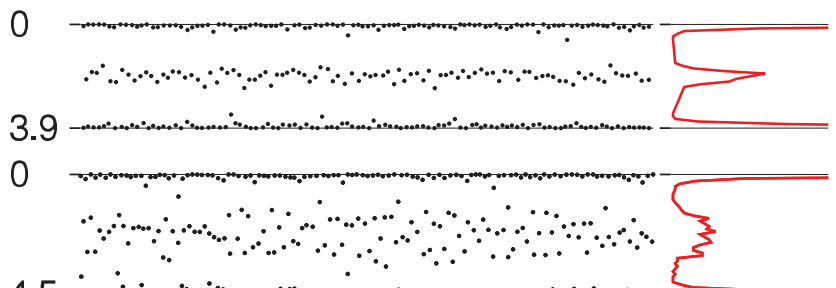

4.5

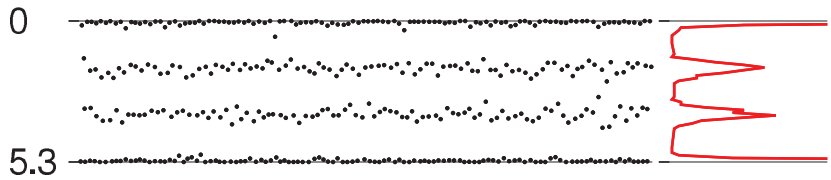

Figure 1 | Typical snapshots from the simulation which show the particle configuration inside the channel. The length of the sections in $x$ direction is $L_{X}^{*}=200$ with a width $\left(L_{Y}^{*}\right)$ of $3.9,4.5$, and 5.3 , respectively. For a better visibility, the snapshots are overstretched in y-direction. The red curves show the density profile along the y-direction averaged over all particles. As it can be seen from these plots, the first and the last channel are ordered in three and four layers, respectively. Around $L_{Y}^{*}=4.5$ the crossover from three to four layer configuration takes place.

displacement (MSD) $\left\langle\Delta x(t)^{2}\right\rangle$ is that of a free Brownian particle (see Figure 2 inset), though it soon evolves into a $\left\langle\Delta x(t)^{2}\right\rangle=2 F \sqrt{t}$ dependence appropriate for single-file motion with $F$ as the singlefile mobility ${ }^{23,24}$. For channel width $L_{Y}^{*}>1$, the long time evolution of the MSD is again expected to be Brownian, since particles can pass each other. The crossover to normal diffusion with increasing channel width is usually described in terms of a hopping time $\tau_{H}$, the average time a particle needs to pass its neighbor. On time scales smaller than $\tau_{H}$ single-file diffusion with an exponent 0.5 , and on time scales greater than $\tau_{H}$ normal diffusion with exponent 1 is observed. For channels with a width $L_{Y}^{*} \rightarrow 1$, the hopping time $\tau_{H}=\infty$.

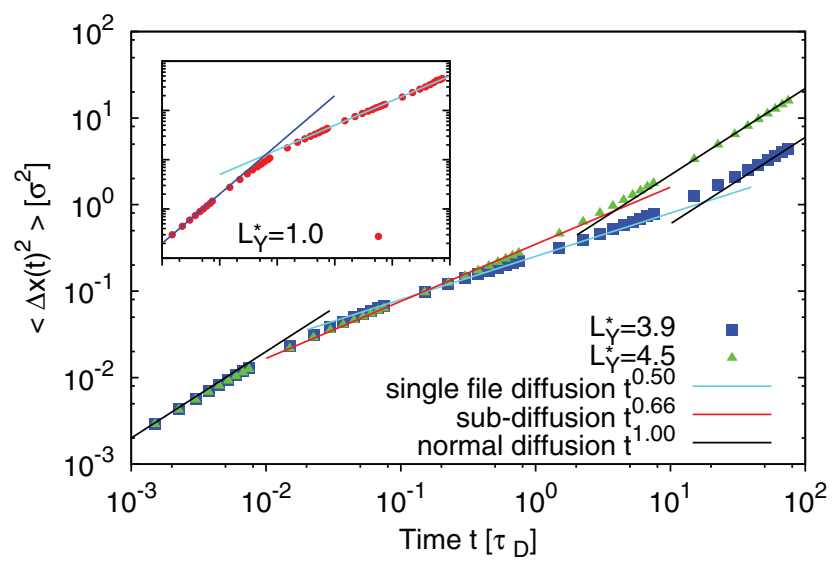

Figure $2 \mid$ Time dependent MSD from the simulation data for selected channel widths in a log-log plot. The diffusion exponent is given by the slope of the curves. The colored solid lines serve as a guideline for the different diffusion exponents and are explained in the legend of the graph. Full rectangles, channel width $L_{Y}^{*}=3.9$, the system is ordered in three layers. Full triangles, channel width $L_{Y}^{*}=4.5$, the system is disordered. In both cases the short and long time diffusion is normal. The intermediate time regime is dominated by the structure inside channel and is single-file like in the ordered case and sub-diffusive in the disordered state. Inset: Time dependent MSD from simulation data (log-log plot) for a channel width $L_{Y}^{*}=1$, the ideal case of single-file diffusion. The diffusion behavior is separated in two regimes, normal diffusion behavior for short times and single-file diffusion in the long time limit.
While for hard spheres, the hopping time $\tau_{H}$ decays exponentially with increasing channel width ${ }^{25}$, our results for the crossover behaviour are influenced by the additional $V \propto 1 / r^{3}$ repulsion arising from the dipole interaction in our system. For $L_{Y}^{*} \gg 1$, this repulsion becomes important, since the dominant restriction for the mutual passing of particles is the layering structure and not hard sphere exclusion. A description using the hopping time is, therefore, problematic for the cross-over phenomenon seen in our studies, since the single-file exponent of $1 / 2$ is reached only in the completely ordered case. Instead, we choose to describe this scenario with an apparent, $L_{Y}$ dependent, intermediate diffusion exponent $\alpha_{\text {inter }}$. We emphasise, $1 / 2 \leq \alpha_{\text {inter }} \leq 1$ is not a new sub-diffusive exponent but rather a convenient way to characterise the time evolution of the MSD $\left(\propto t^{\alpha_{\text {inter }}}\right)$ in an intermediate time regime between the short $(t<$ $\left.0.15 \tau_{D}\right)$ and long time $\left(t>1.5 \tau_{D}\right)$ normal diffusive behaviors suggested by our studies.

We extract values for $\alpha_{\text {inter }}$ by two methods. In the first method, the slope of the MDS data versus time, as shown in Figure 2, is obtained by a linear regression in the region $0.15 \tau_{D}<t<1.5 \tau_{D}$, and this interval has been kept constant for all analysed data, corresponding to the last time decade for the experimental studies before ballistic effects set in, which are not topics of this work. In the second method, the logarithmic derivative of the MSD data, $d\left(\log \left(\left\langle\Delta x(t)^{2}\right\rangle\right)\right) /$ $d(\log (t))$, has been computed by numerical forward difference method as a function of time, see Figure 3 , and the minimum value has been chosen as estimator for $\alpha_{\text {inter }}$. The estimators obtained by both methods are presented in Figure 4 as a function of the channel width, the same analysis method has been applied for the data from the simulations and from the experiment. The minimum values for $\alpha_{\text {inter }}$ obtained by both methods agree fairly well, the maximum values obtained by the regression method are slightly larger compared to the "minimum slope" method due to interval averaging.

We found that even for channel widths $L_{Y}^{*} \gg 1$, for which an integer number of layers fits into the channel, the layering order drives the system back into single-file motion on an intermediate time scale. In Figure 2 the MSD $\left\langle\Delta x(t)^{2}\right\rangle$ is shown in a log-log plot for the three different cases. All MSD curves start with the same shorttime normal diffusive behavior with the microscopic diffusion coefficient $D_{0}$, followed by a sub-diffusive regime. For $L_{Y}^{*}=1$ (Figure 2(inset)) this is already the long-time single-file behavior. For $L_{Y}^{*} \gg 1$ we obtain a sub-diffusive regime with an apparent exponent $0.5<\alpha_{\text {inter }}<1$ as an intermediate behavior. The value of $\alpha_{\text {inter }}$, however, depends crucially on the extent of layer order (Figure 2). If $L_{y}^{*}=3.9$, the particles are ordered into well defined layers (see Figure 1) and we obtain single-file diffusion with $\alpha_{\text {inter }}=1 / 2$ over

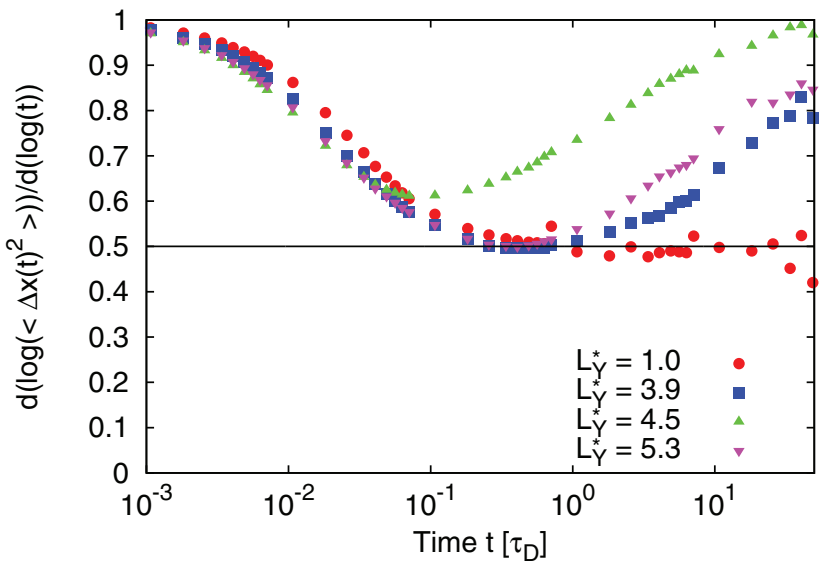

Figure $3 \mid$ Logarithmic derivative of the MSD, $d\left(\log \left(\left\langle\Delta x(t)^{2}\right\rangle\right)\right) / d(\log (t))$, of the simulation data (see Figure 2 ) as a function of time for four values of the channel width. The results for $L_{Y}^{*}=1$ have been computed for the same line density as in the case $L_{Y}^{*}=3.9$. 


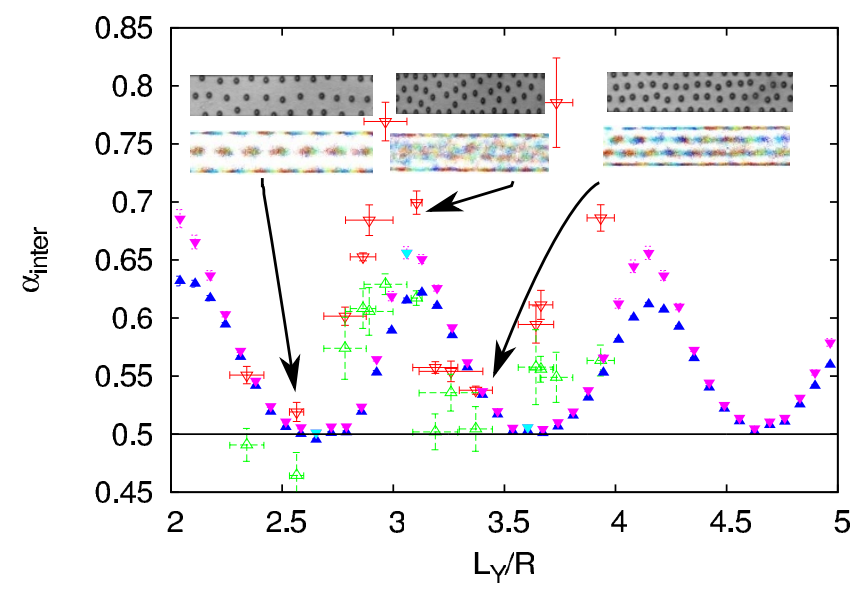

Figure $4 \mid$ The intermediate diffusion exponent $\alpha_{\text {inter }}$ as a function of dimensionless channel width $L_{Y} / R$ for simulation (SIM) and experiment (EXP). The turquois highlighted triangles are the channels depicted in Figure 1. The insets corresponding to the marked $L_{Y} / R$ illustrate the structure inside the channels. The upper part is a snapshot, the lower part shows the particle trajectories over the entire time of the experiment. Open symbols refer to the experiments, full symbols to the simulations, the triangles pointing down refer to the exponent obtained by linear regression to the data in Figure 2 (see text for details), the triangles pointing up refer to the exponent obtained by the minimum slope of the logarithmic derivative of the data (see Figure 3 and text for details). Both experiment and simulation show clearly the non-monotonic dependency of the intermediate exponent on the channel width. For ordered systems it has a value close to the single-file exponent (0.5).

a rather long time-scale spanning more than a decade. On the other hand, for disordered channel structures (e.g. $L_{y}^{*}=4.5$ ), $\alpha_{\text {inter }}$ initially begins to decrease but then approaches the long-time diffusive limit relatively quickly and the $\alpha_{\text {inter }}=1 / 2$ value is never reached.

In Figure 3 we show the logarithmic derivative of the MSD as function of time for various channel widths. A comparison of the data for $L_{Y}^{*}>1$ with the single file diffusion case $\left(L_{Y}^{*}=1\right)$, which has been included for comparison for the same line density as for $L_{Y}^{*}=3.9$, where three well separated layers are present (see Figure 1), reveals that the approach to the single file diffusion exponent $(0.5)$ is achieved only at time scales as large as $\tau_{D}$. At this time scale, particles typically have diffused over a distance of their particle diameter as well and thus can leave their layers in y-direction and enter the region of a neighbouring layer, such that normal diffusion sets in. As a result, the time region, in which the slope of $\log (\mathrm{MSD})$ is minimal, approaching the single file diffusion value, is small, but note again that $\tau_{D}$ is the time region typically limiting the experimental studies. The minimum value for the logarithmic derivative is taken as estimator for $\alpha_{\text {inter }}$ and is shown in Figure 4. From Figure 3 we can conclude that at small times the MSD values for a channel width of $L_{Y}^{*}=1$ are slightly larger compared to the cases with layer formation, since repulsive interaction from particles in neighbouring layers hinder the diffusion in the latter case.

In Figure 4 the intermediate diffusion exponent is plotted as a function of dimensionless channel width $L_{Y} / R$. We see an oscillatory dependency for the simulation results, which is due to the layer structure. If the particles are ordered in layers, the single-file exponent 0.5 can be observed in the intermediate time regime. Otherwise, the MSD is still sub-diffusive in this time regime, but with an exponent greater than the single-file exponent 0.5 .

In experiments it is not possible to vary the channel width. To overcome this problem, one varies the density n, instead. Since the layer spacing $R=(\sqrt{3} / 2 n)^{0.5}$ is the only relevant length scale as long as the particle diameter is small compared to the layer spacing $R / \sigma \ll 1$ and the interaction strength $\Gamma$ is sufficiently large, the variation of the channel width can be compared with a variation of density by introducing the dimensionless channel width $L_{Y} / R$.

The experimental results come from a channel with a width of about $30 \mu \mathrm{m}$. The density in the channel is varied so that the number of layers is between three and four. The mean square displacement is calculated and an intermediate exponent is obtained as in the simulations. One can see a full oscillation of this exponent (Figure 4), while the number of layers vary from a three layers system over a disordered system to a four layer system. The experimental $L_{Y}^{*}$ values for the maxima and minima of $\alpha_{\text {inter }}$ are slightly smaller than the values from the simulations. These differences are caused by the experimental set up, in which several parallel channels are present and a resulting small density reduction at the channel walls due to repulsive interactions with particles from neighboring channels reduces the total density in the channel and thus the value of $L_{Y}^{*} \propto n^{* 1 / 2}$.

\section{Discussion}

In our work we have shown in simulation and experiment that the diffusion of model colloids has a non-monotonic dependency on the channel width. In the experimental accessible region the MSD can be described by a sub-diffusive exponent between 0.5 and 1 , which oscillates with the width of the channel. The effect on the exponent is stable in the experiment and is expected to be general for the diffusion of interacting particles in confined channel geometries. For example, a similar crossover from single-file to normal diffusion has been observed to determine the kinetics of template assisted assembly of colloidal particles ${ }^{26}$.

In general, the diffusion behavior of interacting particles determines the behavior of a large number of systems ranging from pedestrians crossing a road to ions passing through channels in living cells. Our studies of a special system consisting of colloidal particles in confined geometry, accessible both by experimental and theoretical methods, show that the nature of the diffusion process can be modified by changes in the external conditions.

\section{Methods}

In the simulation, a conventional Brownian dynamics algorithm is used to simulate colloidal particles in two-dimensional micro-channels with the pairwise dipole interaction relevant to the experimental system. The finite particle diameter $\sigma$ has been taken into account by a hard-core repulsion. The channel is set up in the $x y$ plane with periodic boundary conditions in $x$-direction and hard wall boundary condition in $y$-direction. Both particle-particle and particle-wall hard-core interaction are realized in a second step as proposed by Heyes ${ }^{21}$.

In the experiment, the particles are trapped in channels made by standard PDMS moulding technique created with SU8 UV-lithography using a master mould ${ }^{17}$. The dimensions of these channels are $2 \mathrm{~mm}$ in length, $30 \mu \mathrm{m}$ in width and $7 \mu \mathrm{m}$ in height, ensuring that the particles are completely confined laterally by the channel walls.

The channel itself is filled with super-paramagnetic colloids (Dynal M-450, diameter $\sigma=4.5 \mu \mathrm{m}, \rho=1500 \mathrm{~kg} / \mathrm{m}^{2}$ ) suspended in water. Gravity confines the particles down to the substrate surface and buoyancy leads to an effective mass of $m_{\text {eff }}$ $=2.38510^{-14} \mathrm{~kg}$. In that case, the particle motion is limited to two dimensions inside the channel. All experiments in the previous sections were done by means of a standard video microscopy setup. The positions of the particles were tracked using particle tracking software which is able to locate the particles with sub-micron accuracy ${ }^{20}$. The sample is aligned horizontally in the video setup to ensure that the particles undergo only Brownian motion. After that, the system is equilibrated for at least $5 \mathrm{~h}$ before the measurement is started. All particle trajectories contain at least 20000 data points with a time resolution of one frame per second. The mean square displacement is calculated directly from these trajectories.

1. Helbing, D., Molnar, P., Farkas, I. \& Bolay, K. Self-organizing pedestrian movement. Environment and Planning B: Planning and Design 28, 361 (2001).

2. Dreher, M., Pauly, F., Heurich, J., Cuevas, J. C., Scheer, E. \& Nielaba, P. Structure and conductance histogram of atomic-sized Au contacts. Phys. Rev. B 72, 075435 (2005).

3. Pauly, F., Viljas, J. K., Bürkle, M., Dreher, M., Nielaba, P. \& Cuevas, J. C. Molecular dynamics study of the thermopower of Ag, Au, and Pt nanocontacts. Phys. Rev. B 84, 195420 (2011).

4. Roth, R. \& Gillespie, D. Physics of Size Selectivity. Phys. Rev. Lett. 95, 247801 (2005). 
5. Squires, T. M. \& Quake, S. R. Microfluidics: Fluid physics at the nanoliter scale. Rev. Mod. Phys. 77, 977 (2005).

6. Köppl, M., Henseler, P., Erbe, A., Nielaba, P. \& Leiderer, P. Layer Reduction in Driven 2D-Colloidal Systems through Microchannels. Phys. Rev. Lett. 97, 208302 (2006).

7. Henseler, P., Erbe, A., Köppl, M., Leiderer, P. \& Nielaba, P. Density reduction and diffusion in driven two-dimensional colloidal systems through microchannels. Phys. Rev. E 81, 041402 (2010).

8. Leunissen, M. E., Christova, C. G., Hynninen, A. P., Royall, C. P., Campbell, A. I., Imhof, A., Dijkstra, M., v. Roij, R. \& v. Blaaderen, A. Ionic colloidal crystals of oppositely charged particles. Nature (London) 437, 235 (2005).

9. Wei, Q. H., Bechinger, C. \& Leiderer, P. Single-File Diffusion of Colloids in OneDimensional Channels. Science 287, 625 (2000).

10. v. Blaaderen, A. Colloidal Molecules and Beyond. Science 301, 470 (2003).

11. Poon, W. Colloids as Big Atoms. Science 304, 830 (2004).

12. Tkalec, U., Ravnik, M., Copar, S., Zumer, S. \& Musevic, I. Reconfigurable Knots and Links in Chiral Nematic Colloids. Science 333, 62 (2011).

13. Means, J. C. \& Wijayaratne, R. Role of Natural Colloids in the Transport of Hydrophobic Pollutants. Science 215, 968 (1982).

14. Kubo, R. Brownian Motion and Nonequilibrium Statistical Mechanics. Science 233, 330 (1986).

15. Han, Y., Alsayed, A. M., Nobili, M., Zhang, J., Lubensky, T. C. \& Yodh, A. G. Brownian Motion of an Ellipsoid. Science 314, 626 (2006).

16. Li, T., Kheifets, S., Medellin, D. \& Raizen, M. G. Measurement of the Instantaneous Velocity of a Brownian Particle. Science 328, 1673 (2010).

17. Xia, Y. \& Whitesides, G. M. Soft Lithography. Angewandte Chemie International Edition 37, 550 (1998).

18. Zahn, K., Lenke, R. \& Maret, G. Two-Stage Melting of Paramagnetic Colloidal Crystals in Two Dimensions. Phys. Rev. Lett. 82, 2721 (1999).

19. Kreuter, C., Leiderer, P. \& Erbe, A. Determination of potential landscapes using video microscopy. Colloid and Polymer Science 290, 575 (2012).

20. Crocker, J. C. \& Grier, D. G. Methods of digital video microscopy for colloidal studies. J. Colloid Interface Science 179, 298 (1996).

21. Heyes, D. M. \& Melrose, J. R. Brownian dynamics simulations of model hardsphere suspensions. J. Non-Newtonian Fluid Mechanics 46, 1 (1993).
22. Haghgooie, R. \& Doyle, P. S. Structural analysis of a dipole system in twodimensional channels. Phys. Rev. E 70, 061408 (2004).

23. Fedders, P. A. Two-point correlation functions for a distinguishable particle hopping on a uniform one-dimensional chain. Phys. Rev. B 17, 40 (1978).

24. Richards, P. M. Theory of one-dimensional hopping conductivity and diffusion. Phys. Rev. B 16, 1393 (1977).

25. Sane, J., Padding, J. T. \& Louis, A. A. Brownian dynamics simulations of model hardsphere suspensions. Faraday Discuss. 144, 285 (2010)

26. Mondal, C. \& Sengupta, S. Single-file diffusion and kinetics of template-assisted assembly of colloids. Phys. Rev. E 85, 020402 (2012).

\section{Acknowledgements}

The authors thank the SFB-TR6 for support and the NIC for computer time. SS acknowledges support from the Indo-EU project MONAMI.

\section{Author contributions}

US performed the $\mathrm{BD}$ simulations, $\mathrm{CK}$ the experiments, $\mathrm{AE}, \mathrm{PL}$ and $\mathrm{PN}$ wrote the text and are responsible for the project planning and the experimental (AE and PL) and theoretical $(\mathrm{PN})$ prerequisites, NS and SS contributed to the theoretical analysis. All authors have discussed and analysed the results and reviewed the manuscript.

\section{Additional information}

Supplementary information accompanies this paper at http://www.nature.com/ scientificreports

Competing financial interests: The authors declare no competing financial interests

License: This work is licensed under a Creative Commons

Attribution-NonCommercial-ShareAlike 3.0 Unported License. To view a copy of this

license, visit http://creativecommons.org/licenses/by-nc-sa/3.0/

How to cite this article: Siems, U. et al. Non-monotonic crossover from single-file to regular diffusion in micro-channels. Sci. Rep. 2, 1015; DOI:10.1038/srep01015 (2012). 